\title{
ORM ASYMPTOTIC EXPANSIONS OF A CLASS OF INTEGRALS IN :MS OF MODIFIED BESSEL FUNCTIONS, WITH APPLICATION TO CONFLUENT HYPERGEOMETRIC FUNCTIONS*
}

\author{
N. M. TEMME†
}

stract. The integral

$$
F_{\lambda}(z, \alpha)=\int_{0}^{\infty} t^{\lambda-1} e^{-z t-\alpha / t} f(t) d t
$$

idered for large values of the real parameter $z ; \alpha$ and $\lambda$ are uniformity parameters in $[0, \infty)$. The otic expansion is given in terms of the modified Bessel function $K_{\lambda}(2 \sqrt{\alpha z})$. The asymptotic nature ixpansion is discussed and error bounds are constructed for the remainders in the expansions. An $\boldsymbol{e}$ is given for confluent hypergeometric or Whittaker functions. In this example the integrals are rmed to standard forms and the mappings are investigated.

y words. uniform asymptotic expansions of integrals, modified Bessel function, confluent hyperric function, Whittaker function, construction of error bounds, transformation to standard form

IS(MOS) subject classifications. 41A60, 30E15, 33A20

- Introduction. We consider integrals of the form

$$
F_{\lambda}(z, \alpha)=\int_{0}^{\infty} t^{\lambda-1} e^{-z t-\alpha / t} f(t) d t,
$$

reduces to a modified Bessel function in the case that $f$ is a constant. We have

$$
2(\alpha / z)^{\lambda / 2} K_{\lambda}(2 \sqrt{\alpha z})=\int_{0}^{\infty} t^{\lambda-1} e^{-z t-\alpha / t} d t .
$$

Itegral in (1.1) is considered with $\alpha, \lambda \geqq 0$ and large positive values of $z$. We aim ive asymptotic expansions for $F_{\lambda}(z, \alpha)$ that hold uniformly with respect to both $\lambda$ in the interval $[0, \infty)$. To handle the transition of the case $\alpha=0$ to $\alpha>0$, the ied Bessel function (1.2) is needed. Observe that when $\alpha=0$ the essential singularthe integrand of (1.1) disappears and that (1.1) becomes a more familiar Laplace al, which can be expanded by using Watson's lemma.

irst we consider fixed values of $\lambda$. To describe the asymptotic features we introduce ssitive number $\beta$ defined by

$$
\beta=\sqrt{\alpha / z} .
$$

zddle points of $\exp (-z t+\alpha / t)$ are located at $t= \pm \beta$. When $\beta$ is bounded away :ero, we can use the familiar Laplace method, since at the point $t=\beta$ the integrand e form of a Gaussian function. When, however, $\alpha \rightarrow 0$, that is, $\beta \rightarrow 0$, the internal $\geq$ point coalesces with the point $t=0$, where the argument of the exponential on has a pole. In addition, there is an algebraic singularity (if $\lambda \neq 1$ ), but the nce of the essential singularity due to the pole is more significant. Observe that limit $\alpha=0$, as mentioned earlier, the pole disappears; also, both saddle points ce with the pole. These asymptotic features are typical for certain integrals $\mathrm{ng}$ Bessel functions. For this reason the modified Bessel function in (1.2) serves

Leceived by the editors May 5, 1988; accepted for publication (in revised form) February 27, 1989.

Zentre for Mathematics and Computer Science, P.O. Box 4079, 1009 AB Amsterdam, the Netherlands. 
as a basic approximant in the uniform asymptotic expansions in this paper. In $\S 4$ we show how an integral with the same phenomena can be transformed into the standard form (1.1).

The integral in (1.1) is the simplest case with the asymptotic features described above, especially when the parameters are in the indicated intervals. We apply the results to a confluent hypergeometric function. By allowing different intervals of integration, say a contour in the complex plane, we can also consider negative values of $\alpha$. Then the ordinary Bessel function $J_{\nu}(z)$ shows up. This case is more difficult, but the applications are very interesting in the theory of special functions.

Consider as an analogue of (1.1) a loop integral in the form

$$
G_{\lambda}(z, \alpha)=\frac{1}{2 \pi i} \int_{-\infty}^{\left(0^{+}\right)} t^{-\lambda-1} e^{z t+\alpha / t} f(t) d t .
$$

This notation means that the contour of integration starts from $-\infty, \arg t=-\pi$, describes a circle counterclockwise around the origin, and returns to $-\infty$, arg $t=+\pi$. The integral (1.4) has the modified Bessel function $I_{\lambda}(2 \sqrt{\alpha z})$ as approximant. When $f=1$ we have

$$
G_{\lambda}(z, \alpha)=(z / \alpha)^{\lambda / 2} I_{\lambda}(2 \sqrt{\alpha z}) .
$$

When $\alpha$ is negative this function is an ordinary $J$-Bessel function. In [2] and [6] integrals of the type (1.4) are treated and the method is used for obtaining a uniform expansion of Laguerre polynomials. We plan to return to this problem in a future paper.

The starting point (1.1) is of interest since it has a real interval of integration. Thus the transformation to the standard form (1.1) involves a real mapping. This makes the first steps of the analysis rather simple, since we do not need to trace the transformed contour in the complex plane. For studying the asymptotic nature of the expansion, we use complex variables, however.

The plan of the paper is as follows. In $\S 2$ we construct a series expansion based on an integration by parts procedure, and we give estimates for the remainder in the expansion. In $\S 3$ we consider an expansion that is based on expanding $f$ at the internal saddle point. In $\S 4$ we give an application to confluent hypergeometric functions. In $\S 5$ the parameter $\lambda$ is considered as a second uniformity parameter in $[0, \infty)$, and again we apply the methods on a confluent hypergeometric function. Especially, we pay attention to the mappings needed for a transformation to the standard form.

Terminology. We call a parameter fixed when it does not depend on the parameters $z, \alpha, \lambda . \Re z=x, \Im z=y$ are the real and imaginary part of $z=x+i y$.

2. An integration by parts procedure. The procedure of this section takes into account both saddle points $\pm \beta$ of the exponential function (where $\beta$ is given in (1.3)), although $-\beta$ lies outside the interval of integration. For this reason we assume that $f$ is also defined at negative values of its argument, and that $f$ is sufficiently smooth for the operations to be used here. Further conditions on $f$ will be given later.

2.1. Construction of the formal series. The first step is the representation

$$
f(t)=a_{0}+b_{0}(t-\beta)+\left(t-\beta^{2} / t\right) g(t),
$$

where $a_{0}, b_{0}$ follow from substitution of $t= \pm \beta$. We have

$$
a_{0}=f(\beta), \quad b_{0}=\frac{1}{2 \beta}[f(\beta)-f(-\beta)] .
$$

Inserting (2.1) into (1.1) we obtain

$$
F_{\lambda}(z, \alpha)=a_{0} A_{\lambda}(z, \beta)+b_{0} B_{\lambda}(z, \beta)+F_{\lambda}^{(1)}(z, \alpha),
$$


where $A_{\lambda}, B_{\lambda}$ are combinations of the modified Bessel functions introduced in (1.2). It is straightforward to verify that

$$
A_{\lambda}(z, \beta)=2 \beta^{\lambda} K_{\lambda}(2 \beta z), \quad B_{\lambda}(z, \beta)=2 \beta^{\lambda+1}\left[K_{\lambda+1}(2 \beta z)-K_{\lambda}(2 \beta z)\right] .
$$

An integration by parts gives

$$
\begin{aligned}
F_{\lambda}^{(1)}(z, \alpha) & =-\frac{1}{z} \int_{0}^{\infty} t^{\lambda} g(t) d \exp \left(-z\left(t+\beta^{2} / t\right)\right) \\
& =\frac{1}{z} \int_{0}^{\infty} t^{\lambda-1} \exp \left(-z\left(t+\beta^{2} / t\right)\right) f_{1}(t) d t
\end{aligned}
$$

with

$$
f_{1}(t)=t^{1-\lambda} \frac{d}{d t}\left[t^{\lambda} g(t)\right]=\lambda g(t)+g^{\prime}(t) .
$$

We see that $z F_{\lambda}^{(1)}(z, \alpha)$ is of the same form as $F_{\lambda}(z, \alpha)$. The above procedure can now be applied to $z F_{\lambda}^{(1)}(z, \alpha)$, and we obtain for (1.1) the formal expansion

$$
F_{\lambda}(z, \alpha) \sim A_{\lambda}(z, \beta) \sum_{s=0}^{\infty} a_{s} z^{-s}+B_{\lambda}(z, \beta) \sum_{s=0}^{\infty} b_{s} z^{-s} \text { as } z \rightarrow \infty
$$

where we define inductively $f_{0}=f, g_{0}=g$ and for $s=1,2, \cdots$

$$
\begin{aligned}
& f_{s}(t)=t^{1-\lambda} \frac{d}{d t}\left[t^{\lambda} g_{s-1}(t)\right]=a_{s}+b_{s}(t-\beta)+\left(t-\frac{\beta^{2}}{t}\right) g_{s}(t), \\
& a_{s}=f_{s}(\beta), \quad b_{s}=\frac{1}{2 \beta}\left[f_{s}(\beta)-f_{s}(-\beta)\right] .
\end{aligned}
$$

Remark 2.1. As mentioned earlier, for this procedure we need function values of $f$ and derivatives at negative values, although the integral (1.1) is defined only for $t$-values in $[0, \infty)$. When we consider analytic functions $f$, as we do later, we assume that $f$ is analytic in a domain $\Omega$ in the complex plane that contains the real line. When, however, $f$ is supposed to belong to $C^{k}[0, \infty)$, we assume in the above procedure that $f$ has been smoothly continued on $(-\infty, 0]$.

2.2. The remainder of the expansion. We introduce a remainder for the expansion in (2.3) by writing

$$
F_{\lambda}(z, \alpha)=A_{\lambda}(z, \beta) \sum_{s=0}^{n-1} a_{s} z^{-s}+B_{\lambda}(z, \beta) \sum_{s=0}^{n-1} b_{s} z^{-s}+z^{-n} R_{n},
$$

where $n=0,1, \cdots$. When $n=0$ the sums are empty and $R_{0}=F_{\lambda}(z, \alpha)$. The integration by parts procedure yields for $R_{n}$ the representation

$$
R_{n}=\int_{0}^{\infty} t^{\lambda-1} \exp \left(-z\left(\frac{t+\beta^{2}}{t}\right)\right) f_{n}(t) d t
$$

where $f_{n}$ is defined by (2.4).

When a bound for $\left|f_{n}(t)\right|$ is available, say,

$$
\left|f_{n}(t)\right| \leqq M_{n}, \quad t \geqq 0, \quad n=0,1, \cdots,
$$

then a bound for $R_{n}$ reads

$$
\left|R_{n}\right| \leqq M_{n} A_{\lambda}(z, \beta)
$$


Since $f_{n}$ depends on $\beta$, the quantity $M_{n}$ may also depend on $\beta$. It follows that for bounded values of $\beta$, say $\beta \in\left[0, \beta_{0}\right], \beta_{0}$ fixed and finite, the estimate (2.8) of the remainder $R_{n}$ shows the asymptotic nature of the expansion $(2.5)$, provided that $(2.7)$ is satisfied.

We must point out that, in general, it is rather difficult to find realistic numbers $M_{n}$ in order to obtain sharp estimates in (2.8). Also, the estimate in (2.7) is rather global, since it takes into account values of $f_{n}$ in the complete interval $[0, \infty)$.

A sharper and more realistic bound for $R_{n}$ may be obtained as follows. Let

$$
w_{\sigma}(t)=\exp \left\{\sigma\left(t+\beta^{2} / t-2 \beta\right)\right\}, \quad t>0, \quad \sigma \geqq 0 .
$$

Observe that $w_{\sigma}(\beta)=1$ and that when $\sigma>0$

$$
\lim _{t \rightarrow 0} w_{\sigma}(t)=\lim _{t \rightarrow+\infty} w_{\sigma}(t)=+\infty \text {. }
$$

We assume that we can assign quantities $\sigma_{n}$ and $M_{n}$, which may depend on $\beta$ and which satisfy

$$
\sigma_{n} \geqq 0, \quad M_{n} \geqq 1+\varepsilon_{n}, \quad \varepsilon_{n} \text { fixed and positive },
$$

such that for all $t>0$ we have

$$
\left|f_{n}(t)\right| \leqq M_{n}\left|f_{n}(\beta)\right| w_{\sigma_{n}}(t) .
$$

Then instead of (2.8) we obtain

$$
\left|R_{n}\right| \leqq M_{n}\left|f_{n}(\beta)\right| \tilde{A}_{\lambda}(z, \beta), \quad z>\sigma_{n},
$$

where

$$
\tilde{A}_{\lambda}(z, \beta)=A_{\lambda}\left(z-\sigma_{n}, \beta\right) e^{-2 \beta \sigma_{n}} .
$$

When $f_{n}(\beta)=0$ a slight modification is needed. The idea about this approach is that in (2.11) function values outside a neighborhood of $t=\beta$ may be estimated very roughly, and that the integral, which results after inserting the right-hand side of (2.11) into (2.6), can be written in terms of one of the approximants in front of the series in (2.5).

A possible approach to computing $M_{n}$ and $\sigma_{n}$ of (2.11) is to start with trial values of $M_{n}$ satisfying (2.10). Then we compute

$$
\sigma_{n}=\sup _{t \geqq 0} \tilde{f}_{n}(t), \quad \beta \text { fixed in }[0, \infty),
$$

where

$$
\tilde{f}_{n}(t)=\frac{\ln \left|f_{n}(t) /\left[M_{n} f_{n}(\beta)\right]\right|}{t+\beta^{2} / t-2 \beta}, \quad t \neq \beta, \quad f_{n}(\beta) \neq 0 .
$$

Observe that the function defined in (2.13) satisfies

$$
\tilde{A}_{\lambda}(z, \beta) / A_{\lambda}(z, \beta)=1+o(1) \text { as } z \rightarrow \infty,
$$

uniformly with respect to $\beta \in[0, \infty)$. This follows from (2.2) and well-known asymptotic relations for the Bessel function.

3. Expansion at the internal saddle point. In the expansion (2.3) we have used function values of $f$ at the negative saddle point $-\beta$. These values appear in the coefficients $a_{s}, b_{s}$ of the expansion. The form of the expansion is very attractive, since only two special functions arise, and also since the parameters $\beta$ and $z$ are nicely 
separated in both series. Although the expansion (2.3) has a canonical form, there remains the drawback that the function $f$ must be defined at $(-\infty, 0]$ in order to obtain for $\beta$ a uniformity domain $[0, \infty)$. For example, it is not possible to obtain such a uniformity domain when $f(t)=1 /(t+1)$. In this section we only expand the function $f$ at the internal saddle point, and we formulate further conditions on $f$ in order to obtain an optimal domain for $\beta$.

3.1. The functions $Q_{s}(\zeta)$ and $\tilde{Q}_{s}(\zeta)$. We expand $f$ in the form

$$
f(t)=\sum_{s=0}^{\infty} a_{s}(\beta)(t-\beta)^{s}, \quad a_{s}=\frac{f^{(s)}(\beta)}{s !} .
$$

Substituting (3.1) in (1.1), we obtain after interchanging the order of summation and integration the formal result

$$
F_{\lambda}(z, \alpha) \sim z^{-\lambda} \sum_{s=0}^{\infty} a_{s}(\beta) Q_{s}(\zeta) z^{-s}, \quad \text { as } z \rightarrow \infty,
$$

where

$$
\begin{gathered}
Q_{s}(\zeta)=\zeta^{\lambda+s} \int_{0}^{\infty} t^{\lambda-1}(t-1)^{s} e^{-\zeta(t+1 / t)} d t, \\
\zeta=\beta z .
\end{gathered}
$$

The functions $Q_{s}(\zeta)$ can be expressed in terms of the modified Bessel functions defined in (1.2). It is easily verified that

$$
Q_{s}(\zeta)=2 \zeta^{\lambda+s} \sum_{r=0}^{s}(-1)^{s-r}\left(\begin{array}{l}
s \\
r
\end{array}\right) K_{\lambda+r}(2 \zeta) .
$$

On the other hand, integrating by parts in (3.3), we obtain the recursion relation

$$
Q_{s+2}=(s+\lambda+1-2 \zeta) Q_{s+1}+\zeta(2 s+\lambda+1) Q_{s}+s \zeta^{2} Q_{s-1}, \quad s=0,1,2, \cdots
$$

For proving the asymptotic properties of (3.2) it is useful to introduce the functions

$$
\tilde{Q}_{s}(\zeta)=\zeta^{\lambda+s} \int_{0}^{\infty} t^{\lambda-1}|t-1|^{s} e^{-\zeta(t+1 / t)} d t .
$$

By applying Laplace's method it is found that for large positive values of $\zeta$

$$
\tilde{Q}_{s}(\zeta) \sim \zeta^{\lambda+(s-1) / 2} e^{-2 \zeta} \Gamma\left(\frac{s+1}{2}\right), \quad s=0,1,2, \cdots .
$$

Furthermore, we have when $z$ is fixed

$$
\lim _{\beta \rightarrow 0} \tilde{Q}_{s}(\zeta)=\Gamma(\lambda+s)
$$

3.2. Error bounds and interpretation of the expansion. We introduce a remainder in the expansion (3.2) by writing

$$
f(t)=\sum_{s=0}^{n-1} a_{s}(\beta)(t-\beta)^{s}+R_{n}(t, \beta)(t-\beta)^{n}, \quad n=0,1,2, \cdots .
$$

Then we obtain for (3.2)

$$
F_{\lambda}(z, \alpha)=z^{-\lambda}\left[\sum_{s=0}^{n-1} a_{s}(\beta) Q_{s}(\zeta) z^{-s}+E_{n}(z, \alpha) z^{-n}\right],
$$


where

$$
E_{n}(z, \alpha)=z^{\lambda+n} \int_{0}^{\infty} t^{\lambda-1}(t-\beta)^{n} R_{n}(t, \beta) \exp \left(-z\left(\frac{t+\beta^{2}}{t}\right)\right) d t
$$

Let $f$ be analytic in a connected domain $\Omega$ of the complex plane; $\Omega$ may depend on $\beta$, and we assume that the radius of convergence $R_{\beta}$ of the expansion (3.1) satisfies the condition

$$
R_{\beta} \geqq \rho(1+\beta)^{\kappa}, \quad \beta \geqq 0 \quad\left(\rho, \kappa \text { fixed, } \rho>0, \kappa \geqq \frac{1}{2}\right) .
$$

This condition says that the distance between the singularities of $f$ and the point $t=\beta$ should be of order $\mathcal{O}\left(\beta^{\kappa}\right)$, uniformly with respect to $\beta \in[0, \infty)$. When $\kappa<\frac{1}{2}$ the singularities of $f$ are too close to the saddle point. Furthermore, we assume that $f$ has the following growth condition in $\Omega$ : there is a real fixed number $p$ such that

$$
\sup _{t \in \Omega}(1+|t|)^{-p}|f(t)|
$$

is bounded for $\beta \in[0, \infty)$.

The coefficients $a_{s}(\beta)$ of $(3.1)$ can be written as

$$
a_{s}(\beta)=\frac{1}{2 \pi i} \int_{C_{r}} \frac{f(t)}{(t-\beta)^{s+1}} d t,
$$

where $C_{r}$ is a circle with centre $\beta$ and radius $r(1+\beta)^{\kappa} ; r$ may depend on $\beta$, but should be uniformly bounded away from zero and small enough to keep $C_{r}$ inside $\Omega$. Using (3.14) we obtain the following form of Cauchy's inequality

$$
\left|a_{s}(\beta)\right| \leqq r^{-s} M_{r}(\beta)(1+\beta)^{-s \kappa},
$$

where

$$
M_{r}(\beta)=\sup _{t \in C_{r}}|f(t)| .
$$

In the next theorem we introduce an asymptotic sequence $\left\{\phi_{s}\right\}$, which is constructed on the basis of the estimates in (3.7) and (3.15). For the concept of asymptotic scale and (generalized) asymptotic expansion we refer to [4, p. 25].

THEOREM 3.1. Let $\zeta=\beta z, \kappa \geqq \frac{1}{2}$, and let

$$
\phi_{s}=\phi_{s}(z, \beta)=M_{r}(\beta)(1+\beta)^{-s \kappa}(1+\zeta)^{\lambda+(s-1) / 2} e^{-2 \zeta} z^{-s}, \quad s=0,1,2, \cdots .
$$

Then $\left\{\phi_{s}\right\}$ is an asymptotic scale as $z \rightarrow \infty$, uniformly with respect to $\beta \in[0, \infty)$.

Proof.

$$
\frac{\phi_{s+1}}{\phi_{s}}=(1+\beta)^{-\kappa} \sqrt{\zeta+1} z^{-1} \leqq \frac{1}{\sqrt{z}} \text { if } z \geqq 1 .
$$

Now we write the expansion (3.2) in the notation

$$
z^{\lambda} F_{\lambda}(z, \alpha) \sim \sum_{s=0}^{\infty} a_{s}(\beta) Q_{s}(\zeta) z^{-s} ; \quad\left\{\phi_{s}\right\} \quad \text { as } z \rightarrow \infty,
$$

and we have the following theorem.

THEOREM 3.2. The expansion (3.19) is a uniform asymptotic expansion as $z \rightarrow \infty$, uniformly with respect to $\beta \in[0, \infty)$. 
Proof. According to the definition of generalized (uniform) asymptotic expansions, we have to prove

$$
z^{-n} E_{n}(z, \beta)=\mathcal{O}\left(\phi_{n}\right), \quad n=0,1,2, \cdots,
$$

as $z \rightarrow \infty$, uniformly with respect to $\beta \in[0, \infty)$. The interval of integration in (3.11) is split up as follows

$$
[0, \infty)=\Delta_{-} \cup\left[t_{-}, t_{+}\right] \cup \Delta_{+},
$$

where

$$
\Delta_{-}=\left[0, t_{-}\right], \quad \Delta_{+}=\left[t_{+}, \infty\right), \quad t_{ \pm}=\beta \pm r_{1}(1+\beta)^{\kappa}, \quad 0<r_{1}<r, \quad r_{1} \text { fixed }
$$

with $r$ as in (3.14). When $t_{-}$happens to be negative, we replace it by 0 . For $t \in\left[t_{-}, t_{+}\right]$ we can write

$$
R_{n}(t, \beta)=\frac{1}{2 \pi i} \int_{C_{r}} \frac{f(\tau)}{(\tau-t)(\tau-\beta)^{n}} d \tau,
$$

with $C_{r}$ as in (3.14). If $\tau \in C_{r}$, we have $|\tau-t| \geqq\left(r-r_{1}\right)(1+\beta)^{\kappa}$. Thus we obtain as in (3.15)

$$
\left|R_{n}(t, \beta)\right| \leqq \frac{M_{r}(\beta)(1+\beta)^{-n \kappa}}{r^{n-1}\left(r-r_{1}\right)} .
$$

Hence $z^{\lambda}$ times the integral over $\left[t_{-}, t_{+}\right]$in (3.11) gives a contribution which is bounded by

$$
\begin{gathered}
\frac{z^{\lambda} M_{r}(\beta)(1+\beta)^{-n \kappa}}{r^{n-1}\left(r-r_{1}\right)} \int_{t_{-}}^{t_{+}} t^{\lambda-1}|t-\beta|^{n} \exp \left(-z\left(\frac{t+\beta^{2}}{t}\right)\right) d t \\
=M_{r}(\beta)(1+\beta)^{-n \kappa} z^{-n} \tilde{Q}_{n}(\zeta) \mathcal{O}(1) \quad \text { as } z \rightarrow \infty,
\end{gathered}
$$

uniformly with respect to $\beta \in[0, \infty)$. Using (3.7), (3.8), and (3.17), we conclude that

$$
z^{-n} E_{n}(z, \alpha)=I_{-}+I_{+}+\mathcal{O}\left(\phi_{n}\right) \text { as } z \rightarrow \infty,
$$

uniformly with respect to $\beta \in[0, \infty)$, where $I_{ \pm}$are the contributions to (3.11) from $\Delta_{ \pm}$. For $t \in \Delta_{ \pm}$we write

$$
(t-\beta)^{n} R_{n}(t, \beta)=f(t)-\sum_{s=0}^{n-1} a_{s}(\beta)(t-\beta)^{s},
$$

and the proof is finished when we have shown that

$$
z^{\lambda} \int_{\Delta_{ \pm}} t^{\lambda-1} e^{-z\left(t+\beta^{2} / t\right)} g(t) d t=\mathscr{O}\left(\phi_{n}\right) \quad \text { as } z \rightarrow \infty,
$$

uniformly with respect to $\beta \in[0, \infty)$, where $g(t)$ is $|f(t)|$ or $\left|a_{s}(t-\beta)^{s}\right|(0 \leqq s \leqq n-1)$. In fact, it is possible to prove that

$$
I_{ \pm} \sim 0 ; \quad\left\{\phi_{s}\right\} \quad \text { as } z \rightarrow \infty,
$$

uniformly with respect to $\beta \in[0, \infty)$. That is, $I_{ \pm}$are asymptotically equal to zero with respect to the scale $\left\{\phi_{s}\right\}$. The proof of (3.28) is similar to that given for another type of integral in [5, Lemma 3.3] and will not be repeated here.

The above theorem gives only an order estimate in terms of $\phi_{n}$ for the remainder defined in (3.11) and gives an interpretation of the asymptotic nature of the expansions (3.2) and (3.19). To obtain a numerical upper bound for $E_{n}(z, \lambda)$ we proceed as in 
the previous section. Since $f(t)$ satisfies the growth condition (3.13), it is possible to find numbers $M_{n}, \sigma_{n}$ satisfying (2.10), such that

$$
\left|R_{n}(t, \beta)\right| \leqq M_{n}\left|a_{n}(\beta)\right| w_{\sigma_{n}}(t), \quad 0<t<\infty .
$$

Using this in (3.11), we obtain the bound

$$
\left|E_{n}(z, \lambda)\right| \leqq M_{n}\left|a_{n}(\beta)\right| e^{-2 \beta \sigma_{n}} \tilde{Q}_{n}\left(\zeta-\beta \sigma_{n}\right), \quad z>\sigma_{n} .
$$

When $a_{n}(\beta)$ happens to vanish as a function of $\beta \in[0, \infty)$, this approach needs a slight modification.

4. Application to confluent hypergeometric functions. We start with the confluent hypergeometric function defined by

$$
\Gamma(a) U(a, b, x)=\int_{0}^{\infty} u^{a-1}(1+u)^{b-a-1} e^{-x u} d u .
$$

We consider $a$ as the large parameter and $x$ as a uniformity parameter in $[0, \infty) ; b$ is a fixed real parameter. We take $b \leqq 1$; the relation

$$
U(a, b, x)=x^{1-b} U(a+1-b, 2-b, x)
$$

can be used when $b>1$.

4.1. Transformation to the standard form. First we give a simple intermediate transformation. The function $[u /(u+1)]^{a}$ assumes its maximal value $($ on $[0, \infty))$ at $u=\infty$. This function controls the asymptotic behaviour of the integrand and, hence, we transform it to an exponential function by writing $u /(u+1)=\exp (-w)$. Then (4.1) becomes

$$
\Gamma(a) U(a, 1-\lambda, x)=\int_{0}^{\infty} w^{\lambda-1} \exp \left(-a w-\frac{x}{e^{w}-1}\right) \tilde{f}(w) d w
$$

where

$$
\tilde{f}(w)=\left[\frac{1-e^{-w}}{w}\right]^{\lambda-1} .
$$

We transform (4.2) into (1.1) with the help of the transformation

$$
w+\frac{\nu}{e^{w}-1}=t+\frac{\beta^{2}}{t}+A
$$

where $\nu=x / a$ and $\beta, A$ are to be determined. We compute them on the following condition on the mapping: the critical points of the $w$-function in (4.4) must correspond with the critical points of the $t$-function. Critical points are $\pm w_{0}, \pm t_{0}$, where

$$
t_{0}=\beta, \quad w_{0}=\cosh ^{-1}(1+\nu / 2)=\ln \left(1+\frac{\nu+W_{0}}{2}\right), \quad W_{0}=\sqrt{\nu^{2}+4 \nu}
$$

It follows that

$$
A=-\frac{\nu}{2}, \quad \beta=\frac{w_{0}+\sinh w_{0}}{2}=\frac{1}{2} \ln \left(1+\frac{\nu+W_{0}}{2}\right)+\frac{1}{4} W_{0}
$$

From the simple differential equation

$$
\frac{d \beta}{d \nu}=\frac{1}{4} \sqrt{(\nu+4) / \nu}
$$


and a Taylor expansion of the right-hand side, it follows that $\beta^{2}$ of (4.4) is an analytic function of $\nu$, at least in the disc $|\nu|<4$. Conversely, $\nu$ is an analytic function of $\beta^{2}$ in some neighborhood of the origin. These domains can be extended to domains containing $[0, \infty)$.

With these values of $A, \beta$ the mapping $w \mapsto t$ is regular at $w= \pm w_{0}$ and at $w=0$. In fact it is regular in $\mathbb{R}$ and as a conformal mapping in a large domain $\Omega$ of the complex plane. We have the correspondences

$$
t( \pm \infty)= \pm \infty, \quad t\left( \pm w_{0}\right)= \pm \beta, \quad t(0)=0 .
$$

More details on the mapping are given in the next subsection.

Using transformation (4.4) in (4.2), we arrive at the standard form

$$
F_{\lambda}(z, \alpha)=\Gamma(a) e^{-x / 2} U(a, b, x)=\int_{0}^{\infty} t^{\lambda-1} e^{-z t-\alpha / t} f(t) d t
$$

with $z=a, \alpha=z \beta^{2}, \lambda=1-b, \beta$ defined in (4.6) with $\nu=x / a$, and

$$
f(t)=\left(\frac{1-e^{-w}}{t}\right)^{\lambda-1} \frac{d w}{d t}, \quad \frac{d w}{d t}=\left(\frac{e^{w}-1}{t}\right)^{2} \frac{t^{2}-\beta^{2}}{\left(e^{w}-1\right)^{2}-\nu e^{w}} .
$$

The function $t(w)$ defined in (4.4) is an odd function of $w$. This easily follows from rewriting (4.4) in the form

$$
\frac{1}{2} \nu+w+\frac{\nu}{e^{w}-1}=t+\frac{\beta^{2}}{t}
$$

After these preparations the expansion of (3.2) can be constructed. The expansion holds uniformly with respect to $\beta \in[0, \infty)$; that is, uniformly with respect to $x \in[0, \infty)$.

The asymptotic nature of the expansion follows from combining (3.20) and (3.17). For this particular case we can derive an upper bound for $M_{r}(\beta)$ of (3.16). The $t$-values on the circle are written as $t=\beta+\tau \sqrt{\beta+1}$, with $|\tau|=r, r$ fixed. When $\beta$ and $\nu$ are large, we derive from (4.6) $\beta=\nu / 4+\ln \sqrt{\nu}+\mathcal{O}(1)$. So, for large values of $\beta$, we obtain (using (4.10)) $t+\beta^{2} / t-\nu / 2=w+\nu /\left(e^{w}-1\right)=\ln \nu+\tau^{2}+\mathcal{O}(1)$. That is, $w \sim \ln \nu$. Then it follows from (4.9) that $f(t)=\mathcal{O}\left(\beta^{1 / 2-\lambda}\right), t \in C_{r}$. Consequently, we can find a fixed number $K$, such that

$$
M_{r}(\beta) \leqq K(\beta+1)^{1 / 2-\lambda}, \quad \beta \in[0, \infty) .
$$

To conclude this subsection, we give the first coefficient $a_{0}(\beta)$ of (3.2). A few calculations based on (4.9) and l'Hôpital's rule yield

$$
\left.\frac{d w}{d t}\right|_{t= \pm \beta}=\sqrt{2 \tanh \left(w_{0} / 2\right) / \beta} .
$$

So we obtain

$$
a_{0}(\beta)=\sqrt{2 \tanh \left(w_{0} / 2\right) / \beta}\left(\frac{1-e^{-w_{0}}}{\beta}\right)^{\lambda-1}
$$

4.2. Analytical properties of the mapping (4.4). We now consider the mapping (4.4) in more detail. We restrict $w$ to the strip

$$
H=\{w \mid \Re w \in \mathbb{R}, \Im w \in[-\pi, \pi]\},
$$

and we prove the following.

THEOREM 4.1. Let $\Omega$ be the image of $H$ under the mapping $w \mapsto t$ defined in (4.4). Let $\nu \in[0, \infty)$ and let $A, \beta$ be defined by (4.6). Then $t(w, \beta)$ is analytic in $H$. 
In the following proof we show that $t(w, \beta)$ and $w(t, \beta)$ are analytic in a fixed neighborhood of $(0,0)$. Accordingly, we concentrate on small (complex) values of the parameters. For remaining values the proof is much easier. For instance, when $\beta$ is bounded away from zero, the critical points $t= \pm \beta$ and the pole at $t=0$ of the right-hand side of (4.4) are well separated. The preparations for applying the Implicit Function Theorem mentioned below are more straightforward then.

Proof. From (4.6) it follows that

$$
w_{0}=\beta[1+o(1)], \quad \nu=\beta^{2}[1+o(1)] \quad \text { as } \beta \rightarrow 0 .
$$

Recall that $t(w)$ is an odd function of $w(\operatorname{see}(4.10))$. We introduce a function $y=y(w, \beta)$ by writing

$$
t=w\left[\frac{\beta}{w_{0}}+\left(w^{2}-w_{0}^{2}\right) y\right] .
$$

This matches the points $w=0 \Leftrightarrow t=0$ and also the critical points $w= \pm w_{0} \Leftrightarrow t= \pm \beta ; y$ is an even function of $w$ and should vanish with $\beta$. Substituting (4.14) in (4.10), we obtain

$$
\frac{\nu t}{w} \frac{\phi(w)-\phi\left(w_{0}\right)}{w^{2}-w_{0}^{2}}+\frac{\beta}{w_{0}}\left(1-\frac{\beta}{w_{0}}\right)+\left(w^{2}-w_{0}^{2}\right)\left(1-\frac{2 \beta}{w_{0}}\right) y-w^{2}\left(w^{2}-w_{0}^{2}\right)^{2} y^{2}=0,
$$

where $\phi(w)=w /(\exp w-1)-1+w / 2$. We expand

$$
\frac{\phi(w)-\phi\left(w_{0}\right)}{w^{2}-w_{0}^{2}}=\sum_{s=1}^{\infty} b_{s}\left(w^{2}-w_{0}^{2}\right)^{s-1}, \quad b_{1}=\frac{\beta-w_{0}}{\nu w_{0}} .
$$

Since $\phi(w)$ is analytic if $|w|<2 \pi$, the series converges if $\beta$ and $w$ are small. Finally, we obtain the equation $F(y, w, \beta)=0$, where $F$ is given by

$$
\frac{\nu \beta}{w_{0}} \sum_{s=2}^{\infty} b_{s}\left(w^{2}-w_{0}^{2}\right)^{s-2}+\nu y \sum_{s=1}^{\infty} b_{s}\left(w^{2}-w_{0}^{2}\right)^{s-1}+\left(1-\frac{2 \beta}{w_{0}}\right) y-w^{2} y^{2} .
$$

The series represents analytic functions of $w, w_{0}$. When $\beta$ is small, $w_{0}$ is an analytic function of $\beta$ (see (4.6)). Hence, $F$ is analytic in a fixed neighborhood of $(0,0,0)$, $F(0,0,0)=0$, and $F_{y}(0,0,0)=-1$. After these preparations we can use an Implicit Function Theorem (see, for instance, [1, p. 36]) and solve for $y(w, \beta)$; it is analytic in a fixed neighborhood of $(0,0)$. By using (4.14) it follows that the same holds for $t(w, \beta)$.

The first terms in the expansion

$$
t(w, \beta)=c_{1}(\beta) w+c_{3}(\beta) w^{3}+\cdots
$$

easily follow from (4.10). We have

$$
c_{1}(\beta)=\frac{\beta^{2}}{\nu}, \quad c_{3}(\beta)=\frac{c_{1}^{2}+\frac{\beta^{2}}{6}-c_{1}-\frac{\nu c_{1}}{4}}{\nu} .
$$

THEOREM 4.2. The mapping (4.4) is univalent in $H$.

Proof. First we show that the mapping is univalent on

$$
\mathscr{L}_{+}=\{w=u+i v \mid u \in \mathbb{R}, v=\pi\},
$$

which is the upper part of the boundary $\partial H$ of $H$. We write $t=r e^{i \theta}$. The image of $\mathscr{L}_{+}$ in the $t$-plane is defined by the equations

$$
\pi=r \sin \theta\left(1-\frac{\beta^{2}}{r^{2}}\right), \quad \Psi(u)=\Phi(\theta)
$$


where

$$
\Psi(u)=\nu / 2+u-\nu /\left(e^{u}+1\right), \quad \Phi(\theta)=r \cos \theta\left(1+\frac{\beta^{2}}{r^{2}}\right) .
$$

The first equation in (4.15) defines a curve given by

$$
r(\theta)=\frac{\pi+\sqrt{\pi^{2}+4 \beta^{2} \sin ^{2} \theta}}{2 \sin \theta}, \quad 0<\theta<\pi .
$$

It follows that $r>\pi / \sin \theta$. Furthermore, we have

$$
\Psi(-\infty)=\Phi(\pi)=-\infty, \quad \Psi(+\infty)=\Phi(0)=+\infty .
$$

The function $\Psi(u)$ is one-to-one on $\mathbb{R}$. The same is true for $\Phi(\theta)$ on $(0, \pi)$, but the proof requires a little extra work. We have, using the first equation in (4.15),

$$
\frac{d r}{d \theta}=-\frac{r \cos \theta\left(r^{2}-\beta^{2}\right)}{\sin \theta\left(r^{2}+\beta^{2}\right)} \text {. }
$$

It follows that

$$
\frac{d \Phi(\theta)}{d \theta}=-\frac{\sin \theta\left(r^{2}+\beta^{2}\right)}{r^{3}}\left[r^{2}+\left(\frac{d r}{d \theta}\right)^{2}\right],
$$

which shows that $\Phi(\theta)$ is one-to-one on $(0, \pi)$. We infer that for each value $u \in \mathbb{R}$ we can find one and only one value $\theta \in(0, \pi)$, such that $\Psi(u)=\Phi(\theta)$, and, hence, one and only one value $r(\theta)$. Since $t(w)$ is an odd function of $w$ (see (4.10)), the mapping $w \mapsto t$ is one-to-one on $\partial H$. When $\Re w \rightarrow \pm \infty$ we have $w \sim t$. Hence the mapping $t(w)$ is also one-to-one as $w \rightarrow \infty, w \in H$. We now consider a large closed rectangle $A B C D$ of which upper side $A B$ and lower side $C D$ are finite parts of $\partial H$, and $B C$ and $A D$ are far away to the right and to the left, respectively. From the above arguments it is not difficult to conclude that the mapping is univalent on $B C, A D$, and on the whole Jordan curve $A B C D A$, provided that the vertical sides are far away. Then we use a well-known result of complex function theory, which says that consequently the mapping is also univalent in the interior of rectangle $A B C D$, since it is analytic there. See [3, Vol. II, p. 118]. We can take the finite rectangle as large as we please. Thus the result also holds for $H$.

For the uniform expansion of (4.8) we take $\Omega$ as the image of the strip $H$ under the mapping $w \mapsto t$. From $f(t)$ defined in (4.9) it follows that (3.13) is bounded in $\Omega$ if $p=1-\lambda$ and that $M_{r}(\beta)$ of (3.16) is well defined. There remains to show that the radius of convergence $R_{\beta}$ of the series in (3.1) satisfies (3.12). It appears that we must take $\kappa=\frac{1}{2}$. In fact, we show that $\Omega$ contains a disc around $\beta$ with radius $\rho \sqrt{\beta+1}(\rho$ fixed), for all $\beta \geqq 0$. The points of intersection of the circle with radius $r$ around the point $t=\beta$ with the curve defined by the first equation of (4.15) are governed by the equations (we write $t=\sigma+i \tau$ )

$$
(\sigma-\beta)^{2}+\tau^{2}=r^{2}, \quad \pi=\tau\left(1-\frac{\beta^{2}}{\sigma^{2}+\tau^{2}}\right), \quad \tau>\pi .
$$

When we require that the circle is tangent at the curve we have the extra condition

$$
\frac{\sigma-\beta}{\tau}=\frac{2 \sigma(\tau-\pi)^{2}}{\pi \beta^{2}+2 \tau(\tau-\pi)^{2}}
$$


This equation is obtained by equating $d \tau / d \sigma$ of both equations and eliminating $\tau^{2}+\sigma^{2}$ by using the second one. For large values of $\beta$ the solution of these three equations reads

$$
\tau=\pi+a \sqrt{\beta}[1+o(1)], \quad \sigma=\beta+b \sqrt{\beta}[1+o(1)], \quad r=c \sqrt{\beta}[1+o(1)],
$$

with $a=b=\sqrt{\pi / 2}, c=\sqrt{\pi}$.

This shows that $\Omega$ is large enough to apply Theorem 3.2. From a further analysis it follows that the value $\kappa=\frac{1}{2}$ is best possible in this case. Apart from the real critical points $\pm w_{0}$ given in (4.5), which are regular points for the mapping, we have other ones located at $\pm w_{0} \pm 2 \pi n i, n=1,2, \cdots$. For large values of $\beta$ those are mapped at a distance $\mathcal{O}(\sqrt{\beta})$ from the critical point $t=\beta$.

Remark 4.1. The behaviour of $f(t)$ of (4.9) in the left half-plane $\mathfrak{R} t<0$ is quite different from that in $\Re t>0$, except when $\lambda=1$. Consequently, the approach of $\S 2$ is less attractive. See also Remark 2.1 .

Remark 4.2. When $b=\frac{1}{2},(4.1)$ is a parabolic cylinder function, and the functions $Q_{s}(\zeta)$ defined in (3.3), (3.5) are elementary functions $\left(\lambda=\frac{1}{2}\right)$. Then (3.2) gives an expansion of the parabolic cylinder function $D_{\nu}(z)$, as $\nu \rightarrow-\infty$, which is uniformly valid with respect to $z \in[0, \infty)$.

5. A second uniformity parameter. In this section we consider (1.1) with $\lambda$ as a second uniformity parameter in $[0, \infty)$. Thus we take further advantage of the fact that the modified Bessel function is a function of two variables. In this case it is convenient to put the reciprocal gamma function in front of the integral. So, now we write

$$
F_{\lambda}(z, \alpha)=\frac{1}{\Gamma(\lambda)} \int_{0}^{\infty} t^{\lambda-1} e^{-z t-\alpha / t} f(t) d t
$$

In [5] we considered (5.1) with $\alpha=0$, again with $z \rightarrow \infty$ and $\lambda$ as a uniformity parameter in $[0, \infty)$. In [6] we applied the present method for a loop integral (without proofs) to the case of Laguerre polynomials.

We write $\lambda=\mu z$. The critical points of the integrand are now defined as the points where the derivative of $t+\beta^{2} / t-\mu \ln t$ vanishes. This gives the real critical points

$$
t_{ \pm}=\frac{\mu \pm T}{2}, \quad T=\sqrt{\mu^{2}+4 \beta^{2}} .
$$

Observe that also in this case one of the real saddle points is outside the interval of integration, and that the "phase function" that is used to compute the critical points has a logarithmic singularity at $t=0$. The two critical points coincide with this singularity when $\beta$ and $\mu$ both vanish. At the same moment, however, the logarithmic singularity disappears.

First we construct an expansion by using the integration by parts procedure of $\S 2$. The modification of $(2.1)$ is

$$
f(t)=c_{0}+d_{0}\left(t-t_{+}\right)+\left(t-\mu-\beta^{2} / t\right) h(t) .
$$

Using this in (5.1) we obtain, after repeating the procedure,

$$
F_{\lambda}(z, \alpha)=C(z, \beta, \mu) \sum_{s=0}^{n-1} c_{s} z^{-s}+D(z, \beta, \mu) \sum_{s=0}^{n-1} d_{s} z^{-s}+z^{-n} R_{n} .
$$

The functions in front of the series are again combinations of Bessel functions as in (2.2). We have

$$
C(z, \beta, \mu)=\frac{2 \beta^{\lambda}}{\Gamma(\lambda)} K_{\lambda}(2 \beta z), \quad D(z, \beta, \mu)=\frac{2 \beta^{\lambda}}{\Gamma(\lambda)}\left[\mu K_{\lambda+1}(2 \beta z)-t_{+} K_{\lambda}(2 \beta z)\right] .
$$


icients $c_{s}, d_{s}$ follow from the recursion relation

$$
\begin{aligned}
& \dot{\mathrm{o}}(t)=f(t), \quad f_{s}(t)=t \frac{d}{d t} h_{s-1}(t)=c_{s}+d_{s}\left(t-t_{+}\right)+\left(t-\mu-\frac{\beta^{2}}{t}\right) h_{s}(t), \\
& \mathrm{s}_{s}=f_{s}\left(t_{+}\right), \quad d_{s}=\frac{f\left(t_{+}\right)-f\left(t_{-}\right)}{t_{+}-t_{-}} .
\end{aligned}
$$

inder $R_{n}$ in (5.4) can be written in the form

$$
R_{n}=\frac{1}{\Gamma(\lambda)} \int_{0}^{\infty} t^{\lambda-1} e^{-z t-\alpha / t} f_{n}(t) d t
$$

can be constructed by using constants $\sigma_{n}, M_{n}$ satisfying (2.10), and using a

$$
w_{\sigma}(t)=\exp \left(\sigma\left(t+\frac{\beta^{2}}{t}-\mu \ln t-t_{+}-\frac{\beta^{2}}{t_{+}}+\mu \ln t_{+}\right)\right)
$$

, as in (2.11), for all $t>0$

$$
\left|f_{n}(t)\right| \leqq M_{n}\left|f_{n}\left(t_{+}\right)\right| w_{\sigma_{n}}(t) .
$$

obtain

$$
\begin{gathered}
\left|R_{n}\right| \leqq M_{n}\left|f_{n}\left(t_{+}\right)\right| \tilde{C}(z, \beta, \mu), \quad z>\sigma_{n}, \\
\tilde{C}(z, \beta, \mu)=C\left(z-\sigma_{n}, \beta, \mu\right) \exp \left(-\sigma_{n}\left(t_{+}+\frac{\beta^{2}}{t_{+}}-\mu \ln \mathrm{t}_{+}\right)\right) .
\end{gathered}
$$

$\left.\boldsymbol{t}_{+}\right)=0$, a slight modification is needed. An optimal value of $\sigma_{n}$ follows from od described in $\S 2.2$.

analogue of the expansion of $\S 3$ is obtained by substituting

$$
f(t)=\sum_{s=0}^{n-1} c_{s}(\beta, \mu)\left(t-t_{+}\right)^{s}+R_{n}(t, \beta, \mu)\left(t-t_{+}\right)^{n}, \quad c_{s}=\frac{f^{(s)}\left(t_{+}\right)}{s !} .
$$

tain

$$
\begin{gathered}
F_{\lambda}(z, \alpha)=z^{-\lambda}\left[\sum_{s=0}^{n-1} c_{s}(\beta, \mu) P_{s} z^{-s}+E_{n}(z, \alpha, \lambda) z^{-n}\right], \\
E_{n}(z, \alpha, \lambda)=z^{\lambda+n} \int_{0}^{\infty} t^{\lambda-1}\left(t-t_{+}\right)^{n} R_{n}(t, \beta, \mu) \exp \left(-z\left(\frac{t+\beta^{2}}{t}\right)\right) d t, \\
P_{s}=\frac{z^{\lambda+s}}{\Gamma(\lambda)} \int_{0}^{\infty} t^{\lambda-1}\left(t-t_{+}\right)^{s} \exp \left(-z\left(\frac{t+\beta^{2}}{t}\right)\right) d t \\
=\frac{2 z^{\lambda+s} \beta^{\lambda}}{\Gamma(\lambda)} \sum_{r=0}^{s}\left(\begin{array}{l}
s \\
r
\end{array}\right)\left(-t_{+}\right)^{s-r} \beta^{r} K_{\lambda+r}(2 \beta z) .
\end{gathered}
$$

ion relation for $P_{s}$ follows from the above integral representation.

$$
\begin{aligned}
\tilde{P}_{s} & =\frac{z^{\lambda+s}}{\Gamma(\lambda)} \int_{0}^{\infty} t^{\lambda-1}\left|t-t_{+}\right|^{s} \exp \left(-z\left(\frac{t+\beta^{2}}{t}\right)\right) d t \\
& \sim \frac{\eta^{\lambda+(s-1) / 2}}{\Gamma(\lambda)} \exp \left(-\eta\left(1+\frac{\beta^{2}}{t_{+}^{2}}\right)\right)\left[\frac{\beta^{2}+t_{+}^{2}}{2 t_{+}^{2}}\right]^{-(s+1) / 2} \Gamma\left(\frac{s+1}{2}\right) \text { as } \eta \rightarrow \infty,
\end{aligned}
$$


where $\eta=z t_{+}$. Since $z$ is the large parameter, $\eta$ is large if at least one of the uniformity parameters $\beta, \mu$ is bounded away from zero.

The coefficients $c_{s}$ and the remainder $R_{n}$ can be written as

$$
c_{s}(\beta, \mu)=\frac{1}{2 \pi i} \int_{C_{r}} \frac{f(\tau)}{\left(\tau-t_{+}\right)^{s}} d \tau, \quad R_{n}(t, \beta, \mu)=\frac{1}{2 \pi i} \int_{C_{r}} \frac{f(\tau)}{(\tau-t)\left(\tau-t_{+}\right)^{n}} d \tau,
$$

where $C_{r}$ is a circle around $t_{+}$with radius $r\left(1+t_{+}\right)^{\kappa}, \kappa \geqq \frac{1}{2}, r>0$. We accept that $f$ depends on both uniformity parameters $\beta, \mu$, and we assume that the domain of analyticity $\Omega$ is large enough to contain such a circle for all $\beta, \mu \geqq 0$.

As in $\S 3$ we have the following theorems. The quantity $M_{r}(\beta, \mu)$ is defined as in (3.16); we also assume that (3.13) is bounded for all $\beta, \mu \in[0, \infty)$.

THEOREM 5.1. Let $\eta=z t_{+}, \kappa \geqq \frac{1}{2}$, and let for $s=1,2, \cdots$

$$
\chi_{s}=\frac{M_{r}(\beta, \mu)}{\Gamma(\lambda) z^{s}} \frac{(1+\eta)^{(s-1) / 2}}{\left(1+t_{+}\right)^{s \kappa}} \exp \left(-\eta\left(1+\frac{\beta^{2}}{t_{+}^{2}}\right)\right)\left[\frac{\beta^{2}+t_{+}^{2}}{2 t_{+}^{2}}\right]^{-(s+1) / 2} .
$$

Then $\left\{\chi_{s}\right\}$ is an asymptotic scale as $z \rightarrow \infty$, uniformly with respect to $\beta, \mu \in[0, \infty)$.

THEOREM 5.2. The expansion

$$
z^{\lambda} F_{\lambda}(z, \alpha) \sim \sum_{s=0}^{\infty} c_{s}(\beta, \mu) P_{s} z^{-s} ; \quad\left\{\chi_{s}\right\} \quad \text { as } z \rightarrow \infty,
$$

is a uniform asymptotic expansion as $z \rightarrow \infty$, uniformly with respect to $\beta, \mu \in[0, \infty)$.

A bound for the remainder $E_{n}$ of (5.6) can be constructed by combining the methods used for (3.30) and the above estimate for the remainder of (5.4).

5.1. Application to a confluent hypergeometric function. Our starting point is (cf.

$$
\begin{aligned}
& \frac{\Gamma(a)}{\Gamma(\lambda)} U(a, 1-\lambda, x) \\
& \quad=\frac{1}{\Gamma(\lambda)} \int_{0}^{\infty} \exp \left(-z\left[-\mu \ln \left(1-e^{-w}\right)+w+\frac{\nu}{e^{w}-1}\right]\right) \frac{d w}{1-e^{-w}},
\end{aligned}
$$

with $z=a, \mu=\lambda / z, \nu=x / z$. The real critical points of the "phase function" are

$$
w_{ \pm}=\ln \left(1+\frac{\mu+\nu \pm W}{2}\right), \quad W=\sqrt{(\mu+\nu)^{2}+4 \nu} .
$$

The transformation to the standard form (5.1) reads

$$
-\mu \ln \left(e^{w}-1\right)+(\mu+1) w+\frac{\nu}{e^{w}-1}=t+\frac{\beta^{2}}{t}-\mu \ln t+A ;
$$

$A, \beta$ are determined by substituting $w_{ \pm}$and $t_{ \pm}$, where $t_{ \pm}$are the critical points defined in (5.2). We have the correspondences

$$
t( \pm \infty)= \pm \infty, \quad t\left(w_{ \pm}\right)=t_{ \pm}, \quad t(0)=0 .
$$

Observe that the introduction of a second parameter (here in the form of $\mu$ ) does not require a third constant in the equation (5.12). It has the same number of constants as (4.4). In fact, in order to obtain a regular mapping $w \mapsto t$, the constants multiplying the log-functions in the left- and right-hand side of (5.12) must be the same. We assume that the log-functions take their principal branches. 
Elimination of $A$ from the two equations (5.12) (with $w=w_{ \pm}, t=t_{ \pm}$) gives a relation for the unknown parameter $\beta$ in terms of $\mu, \nu$ :

$$
(\mu+1) \ln \frac{2+\mu+\nu+W}{2+\mu+\nu-W}-\mu \ln \frac{W+\mu+\nu}{W-\mu-\nu}+W=2 T-\mu \ln \frac{T+\mu}{T-\mu} .
$$

By considering $\mu \in[0, \infty)$ as a fixed parameter, we obtain a more transparent relation for $\beta(\nu)$ in the form of a differential equation:

$$
\frac{d \beta(\nu)}{d \nu}=\frac{\beta W}{2 \nu T}, \quad \beta(0)=0 .
$$

The value of $A$ follows from (5.12) by substituting $w=w_{+}, t=t_{+}$. We have

$$
A=(\mu+1) w_{+}-\mu \ln \frac{\mu+\nu+W}{\mu+T}-\frac{\mu+\nu-W}{2}-T .
$$

Using (5.13), we can eliminate $W / 2-T$ and we obtain

$$
A=\frac{1}{2}\left[(\mu+1) \ln (\mu+1)+\mu \ln \frac{\beta^{2}}{\nu}-\mu-\nu\right] \text {. }
$$

The transformation (5.12) is discussed in the next subsection. By using it in (5.10) we obtain the standard form (5.1):

$$
F_{\lambda}(z, \alpha)=\frac{e^{z A} \Gamma(a)}{\Gamma(\lambda)} U(a, 1-\lambda, x)=\frac{1}{\Gamma(\lambda)} \int_{0}^{\infty} t^{\lambda-1} e^{-z t-\alpha / t} f(t) d t,
$$

where $z=a, \alpha=z \beta^{2} ; \beta^{2}$ follows from (5.13) with $\mu=\lambda / z, \nu=x / z$. Furthermore,

$$
f(t)=\frac{t}{1-e^{-w}} \frac{d w}{d t}=\frac{e^{w}\left(e^{w}-1\right)}{t} \frac{t^{2}-\mu t-\beta^{2}}{\left(e^{w}-1\right)^{2}-(\mu+\nu)\left(e^{w}-1\right)-\nu} .
$$

The first coefficient of (5.9) equals $f\left(t_{+}\right)$. A few computations give

$$
c_{0}(\beta, \mu)=e^{w_{+} / 2} \sqrt{T / W}
$$

The function $f$ satisfies $f(t) \sim t$ as $t \rightarrow+\infty$, whereas $f$ is exponentially small at $-\infty$. This time we can also derive an expansion based on (5.4).

5.2. Analytical properties of the mapping (5.12). The mapping $w \mapsto t$ defined in (5.12) is one-to-one on the strip $H$ given in (4.13). First we prove this property for the boundary. The proof is similar to that for Theorem 4.2. The equations for the image of the upper part of $\partial H$ are given by (cf. (4.15))

$$
\pi=r \sin \theta\left(1-\frac{\beta^{2}}{r^{2}}\right)-\mu \theta, \quad \Psi(u)=\Phi(\theta),
$$

where

$$
\Psi(u)=-A+(\mu+1) u-\frac{\nu}{e^{u}+1}, \quad \Phi(\theta)=r \cos \theta\left(1+\frac{\beta^{2}}{r^{2}}\right)-\mu \ln r .
$$

It follows that the image is given by

$$
r(\theta)=\frac{\mu \theta+\pi+\sqrt{(\mu \theta+\pi)^{2}+4 \beta^{2} \sin ^{2} \theta}}{2 \sin \theta}, \quad 0<\theta<\pi .
$$

The function $\Psi(u)$ is one-to-one on $\mathbb{R}$. When we compute $d \Phi(\theta) / d \theta$, we find the same expression as in (4.16). As in Theorem 4.2, we conclude that the mapping is univalent on the boundary for all $\beta, \mu \in[0, \infty)$. 
It remains to show that the mapping is analytic inside $H$. The interesting question is: Is $t(w)$ analytic at $t=0, t=w_{ \pm}$, uniformly with respect to the parameters $\nu, \mu$ ? Especially interesting are small values of the parameters, since then the critical points coalesce with the pole and log-singularity at $w=0$. When one of the parameters is bounded away from the origin, the critical points $w_{ \pm}$are well separated. In that case the problem is simpler. Here we prove that $t(w, \nu, \mu)$ is analytic for complex values of the three arguments in a fixed neighborhood of $(0,0,0)$. The proof follows the idea of $\S 4.2$.

First we have the following theorem.

THEOREM 5.1. $\beta^{2}=\beta^{2}(\nu, \mu)$ defined by (5.13) is an analytic function of $\nu, \mu$.

Proof. As remarked earlier, we concentrate on small values of the parameters. For $\mu=0$ the relation between $\nu$ and $\beta$ is given in (4.6), and we have mentioned there that $\beta^{2}(\nu, 0)$ is analytic in the domain of interest. On the other hand, we have the expansion

$$
\beta^{2}(\nu, \mu) \sim c_{1}(\mu) \nu+c_{2}(\mu) \nu^{2}+c_{3}(\mu) \nu^{3}+\cdots \quad \text { as } \nu \rightarrow 0 .
$$

The coefficients $c_{s}$ are analytic functions of $\mu$. The first few easily follow from (5.13):

$$
\begin{gathered}
c_{1}(\mu)=e^{(\mu+1) \ln (\mu+1) / \mu-1}=1+\frac{1}{2} \mu-\frac{1}{24} \mu^{2}+\mathcal{O}\left(\mu^{3}\right) \quad \text { as } \mu \rightarrow 0, \\
c_{2}(\mu)=\frac{c_{1}(\mu)\left[\mu+2-2 c_{1}(\mu)\right]}{\mu^{2}}=\frac{1}{12}+\mathcal{O}(\mu) \quad \text { as } \mu \rightarrow 0 .
\end{gathered}
$$

Next we observe that the quantity $T$ of (5.2) is singular at $\beta^{2}=-\mu^{2} / 4$ and that $W$ of (5.11) has singular points at $\nu=\nu_{0}, \nu=\nu_{1}$, where

$$
\nu_{0}=-(\mu+2)+2 \sqrt{\mu+1}, \quad \nu_{1}=-(\mu+2)-2 \sqrt{\mu+1} .
$$

It is obvious that the singularities at $-\mu^{2} / 4, \nu_{0}$ must correspond. That is, a necessary condition for $\beta^{2}$ to be regular for small values of $|\mu|$ is $\beta^{2}\left(\nu_{0}, \mu\right)=-\mu^{2} / 4$. Note that $\nu_{0} \sim-\mu^{2} / 4$ as $\mu \rightarrow 0$ and that (5.13) is satisfied when we substitute $T=W=0$.

We "remove" the singularity at $\nu=\nu_{0}$ from (5.13), and we introduce a function $X=X(q, \mu)$ by writing

$$
\frac{T+\mu}{T-\mu} \frac{W-\mu-\nu}{W+\mu+\nu}=\frac{1+\sqrt{q} X}{1-\sqrt{q} X}, \quad q=\nu-\nu_{0} .
$$

In other words,

$$
\begin{gathered}
\sqrt{q} X=\frac{\mu W-(\nu+\mu) T}{W T-\mu(\mu+\nu)}, \quad T=\mu[W+(\nu+\mu) \sqrt{q} X] / D, \\
D=\nu+\mu+\sqrt{q} X W .
\end{gathered}
$$

Now we can rewrite (5.13) in the form $K+L+M=0$, with

$$
\begin{aligned}
& K=(W-2 T) D=W(\nu-\mu)+\sqrt{q} X\left(\nu^{2}+4 \nu-\mu^{2}\right), \\
& L=D(\mu+1) \ln \frac{2+\mu+\nu+W}{2+\mu+\nu-W}=D(\mu+1) \ln \frac{1+\sqrt{q} Z}{1-\sqrt{q} Z}, \\
& M=D \mu \ln \frac{T+\mu}{T-\mu} \frac{W-\mu-\nu}{W+\mu+\nu}=D \mu \ln \frac{1+\sqrt{q} X}{1-\sqrt{q} X}
\end{aligned}
$$


where

$$
Z=\frac{\sqrt{\nu-\nu_{1}}}{2+\mu+\nu}=\frac{\sqrt{q+\nu_{0}-\nu_{1}}}{q+2+\mu+\nu_{0}} .
$$

We expand $K+L+M$ in powers of $q$. A first observation is that $F(q, X, \mu):=$ $(K+L+M) / \sqrt{q}$ is a function of $q, X, \mu$, the factor $\sqrt{q}$ being completely removed. We expand $F$ in powers of $q$. We have

$$
F(q, X, \mu)=F_{0}+F_{1} q+F_{2} q^{2}+\cdots,
$$

where $F_{s}(X, \mu)$ do not explicitly depend on $q$ (or $\nu$ ). We compute

$$
F_{0}=\left(\nu_{0}-\mu\right) \sqrt{\nu_{0}-\nu_{1}}-2 \mu\left(\nu_{0}+\mu\right) X+\frac{2 \sqrt{\nu_{0}-\nu_{1}}\left(\nu_{0}+\mu\right)(\mu+1)}{2+\mu+\nu_{0}}+2 \mu\left(\nu_{0}+\mu\right) X .
$$

It appears that $F_{0}(X, \mu) \equiv 0$, and that, hence, we can continue with the equation $G(q, X, \mu):=F / q=F_{1}+F_{2} q+\cdots=0$. We claim that the equation $G(q, X, \mu)=0$ can be solved for $X=X(q, \mu)$, and that $X$ is analytic for small values of both arguments. By calculating some limits, it follows from $(5.18)$ or $(5.19)$ that $X(0,0)=-\frac{1}{2}$. This is used to show that $G\left(0,-\frac{1}{2}, 0\right)=F_{1}(0,0)=0$. In order to apply an Implicit Function Theorem (see $[1$, p. 36]), we need to show that $G$ is analytic in a neighborhood of $\left(0,-\frac{1}{2}, 0\right)$ and that $G\left(0,-\frac{1}{2}, 0\right)=0, G_{X}\left(0,-\frac{1}{2}, 0\right) \neq 0$. It is straightforward to verify that $G(q, X, \mu)$ is analytic in a neighborhood of $\left(0,-\frac{1}{2}, 0\right)$. Furthermore, $G_{X}\left(0,-\frac{1}{2}, 0\right)=$ $\partial F_{1} / \partial X=4$ at $(X, \mu)=\left(-\frac{1}{2}, 0\right)$. We have shown that we can solve the equation $G=0$ and that the solution $X(q, \mu)$ is analytic in a fixed neighborhood of $(0,0)$.

It remains to show that $\beta^{2}$ is analytic. We consider $T$ of $(5.2)$ given in the middle of (5.19). We are done when we have shown that $\mu / D$ is bounded away from zero when $\mu$ is small, since then we can divide the denominator of $T$ by $\mu$. From the above result it follows that we can expand

$$
X(q, \mu)=X_{0}(q)+X_{1}(q) \mu+\cdots,
$$

where the coefficients $X_{s}$ are analytic functions of $q$. From the first equation of (5.19) we compute $X_{0}=-1 / \sqrt{\nu-\nu_{1}}=-1 / \sqrt{\nu+4}$. Hence

$$
D=\nu+\mu+\left(\nu-\nu_{0}\right) \sqrt{\nu-\nu_{1}} X_{0}+\mathcal{O}(\mu)=\mathcal{O}(\mu)
$$

as $\mu \rightarrow 0$. It now follows that $T^{2}$ is an analytic function of $q, \mu$ in a fixed neighborhood of $(0,0)$, and, consequently, that $\beta^{2}$ is analytic. This proves the theorem.

Remark 5.1. It is possible to base a proof on the differential equation (5.14). The condition $\beta(0)=0$ is not enough to prove the theorem, since the ratio $\beta^{2} / \nu$ (at $\left.\nu=0\right)$ turns out to be undefined. Requiring that this ratio equals $c_{1}(\mu)$ is sufficient, however.

In Theorem 4.1 we expanded the functions of (4.10) at the critical points $\pm w_{0}$, and in (4.14) we used a representation of $t$ in which $y$ can be viewed as a part of the complete expansion. In fact, (4.14) is a change of variables. In the present case we expand at the critical points $w_{ \pm}$, and the expansions have the form

$$
\psi(w)=\sum_{k=0}^{\infty}\left[a_{k}+w b_{k}\right] V^{k}, \quad V=V(w)=\left(w-w_{-}\right)\left(w-w_{+}\right) .
$$

When $\psi$ is sufficiently smooth, the coefficients $a_{k}, b_{k}$ are uniquely defined. The first 
few are given by

$$
\begin{array}{ll}
a_{0}=\frac{\psi_{-} w_{+}-\psi_{+} w_{-}}{w_{+}-w_{-}}, & b_{0}=\frac{\psi_{+}-\psi_{-}}{w_{+}-w_{-}} \\
a_{1}=\frac{b_{0} w_{0}-\psi_{+}^{\prime} w_{-}-\psi_{-}^{\prime} w_{+}}{\left(w_{+}-w_{-}\right)^{2}}, & b_{1}=\frac{\psi_{+}^{\prime}+\psi_{-}^{\prime}-2 b_{0}}{\left(w_{+}-w_{-}\right)^{2}},
\end{array}
$$

where $w_{0}=w_{+}+w_{-}$, and $\psi_{+}=\psi\left(w_{+}\right)$, etc. For analytic functions the coefficients can be represented as Cauchy-type integrals. We have

$$
a_{k}=\frac{1}{2 \pi i} \int_{C}\left(w-w_{0}\right) V^{-k-1}(w) \psi(w) d w, \quad b_{k}=\frac{1}{2 \pi i} \int_{C} V^{-k-1}(w) \psi(w) d w,
$$

where $C$ is a contour around the two critical points; $\psi$ must be analytic inside $C$ and continuous on $C$. This can be verified by substituting a new variable $w=v+w_{0} / 2$. Then we have

$$
\psi(w)=f\left(v+\frac{w_{0}}{2}\right)=\sum c_{k} V^{k}+v \sum b_{k} V^{k}, \quad c_{k}=a_{k}+\frac{1}{2} w_{0} b_{k} .
$$

By separating odd and even parts (with respect to $v$ ), and representing $c_{k}, b_{k}$ as Cauchy integrals in the $V$-plane, we arrive at (5.21). (Note that a circle around the origin in the $w$-plane is traversed twice in the $V$-plane.) For MacLaurin series the domain of convergence is a disc. For expansions as in (5.20) the domain of convergence is defined by $|V(w)|<\left|V\left(w_{s}\right)\right|$, where $w_{s}$ is a singularity of $\psi$; this domain is bounded by a Cassini's oval with foci at $w_{ \pm}$. See also [7, Exercise 24, p. 149].

The parameter $t$ of (5.12) is represented in the form

$$
t=w[B+C w+V(w) y]
$$

where $B, C$ do not depend on $w$, and we require that the points $\left\{w_{-}, 0, w_{+}\right\}$correspond with $\left\{t_{-}, 0, t_{+}\right\}$. This gives for $B, C$ the values

$$
B=\frac{w_{+}^{2} t_{-}-w_{-}^{2} t_{+}}{w_{+} w_{-}\left(w_{+}-w_{-}\right)}, \quad C=\frac{t_{+} w_{-}-t_{-} w_{+}}{w_{-} w_{+}\left(w_{+}-w_{-}\right)} .
$$

The critical points $w_{ \pm}, t_{ \pm}$are not analytic for small values of the parameters. However, we have the following lemma.

Lemma 5.1. $B, C, w_{+} w_{-}, w_{0}=w_{+}+w_{-}$are analytic functions of $\mu, \nu$ in a fixed neighborhood of $(0,0)$. Moreover, $B=1+o(1), C=o(1)$ near $(0,0)$.

Proof. We use the notation of Theorem 5.1. We have $w_{0}=\ln (1+\mu)$ and the product $w_{+} w_{-}$is an even function of $W$. So the singularity in $W=\sqrt{q} \sqrt{\nu-\nu_{1}}$ is removed when we expand $w_{+} w_{-}$in powers of $W$. Using (5.2), we can write

$$
2 C=-\frac{\mu}{w_{+} w_{-}}\left[1-\frac{T \ln (1+\mu) / \mu}{w_{+}-w_{-}}\right] \text {. }
$$

We introduce a parameter $\eta$ by writing

$$
\beta^{2}=\nu\left[E+\left(\nu-\nu_{0}\right) \eta\right], \quad E=-\frac{\mu^{2}}{4 \nu_{0}}=-\frac{\nu_{1}}{4} .
$$

Then we have $T=2 \sqrt{q} \sqrt{E+\nu \eta} ; \eta=\eta(\nu, \mu)$ is analytic in a neighborhood of $(0,0)$. Next we use $w_{+}-w_{-}=\ln [(1+\sqrt{q} Z) /(1-\sqrt{q} Z)]$. Since the factor $\sqrt{q}$ can be removed, we infer that the fraction $T /\left(w_{+}-w_{-}\right)$is regular. It is easily verified that the expression 
between square brackets in (5.24) vanishes when $\nu \rightarrow 0$ and that $w_{+} w_{-}=-\nu F$, where $F=F(\nu, \mu)$ is analytic at $(0,0)$, with $F=1+\mathcal{O}(\nu+\mu)$, as $\nu, \mu \rightarrow 0$. This proves that $C$ is analytic at $(0,0)$; the factor $\mu$ in the first fraction of (5.24) takes care of the vanishing of $C$ at $(0,0)$. A more detailed analysis shows that $C \sim-\mu / 24, \mu \rightarrow 0, \nu=0$. The proof for $B$ now follows from the representation $B+w_{0} C=\left(t_{+}-t_{-}\right) /\left(w_{+}-w_{-}\right)=$ $T /\left(w_{+}-w_{-}\right)$. At $\nu=0$ this expression reduces to $\mu / \ln (\mu+1)=1+\mathcal{O}(\mu)$, as $\mu \rightarrow 0$.

COROLlARY 5.1. Let $\psi$ of (5.20) be analytic in a domain containing the points $w_{ \pm}$. Then the coefficients $a_{k}, b_{k}$ are analytic functions of the parameters $\mu, \nu$.

Proof. This follows from the fact that sum and product of $w_{ \pm}$occur in $V(w)$ and that the Cauchy-type integrals in (5.21) are analytic functions of $w_{+}+w_{-}$and $w_{+} w_{-}$.

After these preparations we are ready to consider the following theorem.

THEOREM 5.2. The function $t(w, \nu, \mu)$ defined by (5.12), with $\beta^{2}$ defined in (5.13), is analytic in a fixed neighborhood of $(0,0,0)$.

Proof. We write (5.12) in the form

$$
F(t, w, \mu, \nu)=t H(w)-S(t)=0,
$$

where

$$
H(w)=-\mu \ln \frac{e^{w}-1}{w}+(\mu+1) w+\frac{\nu}{e^{w}-1}-A, \quad S(t)=t^{2}+\beta^{2}-\mu t \ln \frac{t}{w} .
$$

Using (5.22) we can consider $F$ as a function of $w$, with two known parameters $\mu, \nu$, and one unknown parameter $y$. We expand $F$ as in (5.20):

$$
F=\sum_{k=0}^{\infty}\left[u_{k}+w v_{k}\right] V^{k}(w)
$$

where the coefficients $u_{k}, v_{k}$ do not depend on $w$ and $t$; they do depend on $y$, however. The first coefficients are

$$
\begin{aligned}
u_{0}=- & C^{2} b^{2} a-C^{2} a^{2}-a B^{2}-2 a B C b+f_{0} B+g_{0} a C-\beta^{2} \\
& +\mu a\left(C c_{0}+B d_{0}+C b d_{0}\right), \\
v_{0}=- & 2 a B C-C^{2} b^{3}-b B^{2}+f_{0} C+g_{0} B-2 C^{2} a b-2 b^{2} B C+g_{0} b C \\
& +\mu\left(C a d_{0}+B c_{0}+C b c_{0}+b B d_{0}+C b^{2} d_{0}\right), \\
u_{1}=- & B^{2}-C^{2} b^{2}-2 a B y-2 C b a y-2 C^{2} a+f_{0} y+f_{1} B-2 B C b+g_{0} C+g_{1} a C \\
& +\mu\left(a B d_{1}+C b a d_{1}+C a c_{1}+C c_{0}+B d_{0}+C b d_{0}+a y d_{0}\right), \\
v_{1}=- & 2 b B y-2 C b^{2} y-2 B C-2 C a y-2 C^{2} b+g_{0} y+f_{1} C+g_{1} B+g_{1} b C \\
& +\mu\left(b B d_{1}+C b^{2} d_{1}+B c_{1}+C a d_{1}+C b c_{1}+C d_{0}+y c_{0}+b y d_{0}\right), \\
u_{2}=- & a y^{2}+g_{1} C+f_{2} B+f_{1} y-2 B y+g_{2} a C-C^{2}-2 C b y \\
& +\mu\left(C a c_{2}+a B d_{2}+B d_{1}+C c_{1}+y d_{0}+C b d_{1}+C b a d_{2}+a y d_{1}\right), \\
v_{2}=g_{2} b C-b y^{2}+g_{1} y-2 C y+g_{2} B+f_{2} C & \\
& +\mu\left(C b^{2} d_{2}+b y d_{1}+C b c_{2}+C a d_{2}+B c_{2}+b B d_{2}+y c_{1}+C d_{1}\right),
\end{aligned}
$$

where $a, b$ are defined by $w^{2}=a+b w+V(w)$, i.e., $a=-w_{+} w_{-}, b=w_{+}+w_{-}$and the coefficients $c_{k}, d_{k}, f_{k}, g_{k}$ occur in the expansions

$$
\begin{aligned}
& w H(w)=f_{0}+g_{0} w+f_{1} V+g_{1} w V+f_{2} V^{2}+g_{2} w V^{2}+\cdots, \\
& \ln \frac{t}{w}=c_{0}+d_{0} w+c_{1} V+d_{1} w V+c_{2} V^{2}+d_{2} w V^{2}+\cdots .
\end{aligned}
$$


The coefficients $u_{0}, v_{0}$ vanish identically. This can be verified by straightforward manipulations. It also follows from the observation that the representation (5.22) can be viewed as a truncated expansion for $t$, in which the first coefficients $B, C$ are defined properly. If more coefficients $D, E, \cdots$ had been included in $y$ (and defined properly), more and more coefficients $u_{k}, v_{k}$ would vanish identically. When using (5.22), only a few coefficients will vanish. Although $u_{1}, v_{1}$ contain the parameter $y$ (also via $c_{1}$, $d_{1}$ ), these coefficients vanish too. Again, this can be verified by straightforward manipulations.

It follows that we can proceed with the equation $G=0$, where

$$
G=G(y, w, \mu, \nu)=\frac{F(t, w, \mu, \nu)}{V^{2}(w)} .
$$

The coefficient $u_{2}$ contains a term $-2 B y$, with $B$ given in (5.23). From Lemma 5.1, it follows that $B$ is bounded away from zero when the parameters $\mu, \nu$ are small. The remaining contributions to $u_{2}$ containing the parameter $y$ tend to zero as $\mu, \nu \rightarrow 0$. All coefficients $u_{k}, v_{k}$ are analytic functions of $\mu, \nu$, and the convergent infinite series (including coefficients $v_{2}$ and higher) represents a function of $y, w, \mu, \nu$ that is analytic in a neighborhood of $(0,0,0,0)$. Consequently, since $\partial G(0,0,0,0) / \partial y=-2$, we can solve for $y$ and this solution is an analytic function of $w, \mu, \nu$ in a fixed neighborhood of $(0,0,0)$. The same holds for $t$ given in (5.22).

Remark 5.3. A simpler version $(\mu=0)$ of the above theorem is considered in Theorem 4.1. Another simpler version $(\nu=0)$ is given by [5, Thm. 2.1].

We still have to show that $\Omega$ (the image of strip $H$ of (4.13) under the mapping $w \mapsto t$ defined in (5.12)) is large enough to contain a disc around $t_{+}$with radius $\rho\left(1+t_{+}\right)^{\kappa}, \kappa \geqq \frac{1}{2}, \rho$ fixed. It is not difficult to verify that when $\beta>\mu$ the proof runs as in $\S 4$.2. If $\mu$ is much larger than $\beta$, the situation improves, and we can take $\kappa=1$.

We conclude by computing a bound for the quantity $M_{r}(\beta, \mu)$ used in (5.8), and defined as in (3.16). The $t$-values on the circle $C_{r}$ are written as $t=t_{+}+\tau \sqrt{t_{+}+1}$, with $|\tau|=r, r$ fixed. We assume that at least one of the parameters $\nu, \mu$ is large. We have

$$
t+\frac{\beta^{2}}{t}-\mu \ln t \sim t_{+}+\frac{\beta^{2}}{t_{+}}-\mu \ln t_{+}+\frac{T\left(1+t_{+}\right)}{t_{+}(\mu+T)} \tau^{2}+\mathcal{O}\left(t_{+}^{-1}\right) .
$$

We denote the factor multiplying $\tau^{2}$ by $q$. Observe that, roughly speaking, $q$ belongs to the interval $\left[\frac{1}{2}, 1\right]$. Using this in $(5.12)$, we obtain

$$
q \tau^{2} \sim-\mu \ln \frac{e^{w}-1}{e^{w_{+}}-1}+(\mu+1)\left(w-w_{+}\right)+\nu\left[\frac{1}{e^{w}-1}-\frac{1}{e^{w_{+}}-1}\right] .
$$

Denoting the right-hand side by $\psi(w)$, we see that $\psi\left(w_{+}\right)=\psi^{\prime}\left(w_{+}\right)=0$. A few computations give

$$
\psi^{\prime \prime}\left(w_{+}\right)=1+\frac{2(\nu+W)}{(\nu+\mu+w)^{2}}=1+o(1) .
$$

To solve the equation $\psi(w)=q \tau^{2}$ we expand $\psi\left(w_{+}+v\right)=\frac{1}{2} v^{2} \psi^{\prime \prime}\left(w_{+}\right)+\cdots$. We can take the fixed number $r$ as small as we please. Then the solution of the above equation reads $w \sim w_{+}+\tau \sqrt{2 q}$. Using this in (5.16), we infer that $f(t) \sim 4 \sqrt{t_{+}} / \tau$, under the condition that $t \in C_{r}$ and that at least one of the parameters $\nu, \mu$ is large. Consequently, we can find a fixed number $K$, such that

$$
M_{r}(\beta, \mu) \leqq K \sqrt{1+t_{+}} / r, \quad \nu, \mu \in[0, \infty) .
$$




\section{REFERENCES}

[1] Sh-N. Chow AND J. K. Hale, Methods of Bifurcation Theory, Springer-Verlag, Berlin, New York, 1982.

[2] C. L. FRENZEN AND R. WONG, Uniform asymptotic expansions of Laguerre polynomials, SIAM J. Math. Anal., 19 (1988), pp. 1232-1248.

[3] A. I. Markushevich, Theory of Functions of a Complex Variable, Prentice-Hall, Englewood Cliffs, NJ, 1965.

[4] F. W. J. Olver, Asymptotics and Special Functions, Academic Press, New York, 1974.

[5] N. M. TEMME, Laplace type integrals: transformation to standard form and uniform asymptotic expansions, Quart. Appl. Math., XLIII (1985), pp. 103-123.

[6] - Laguerre polynomials: asymptotics for large degree, Report AM-R8610, Centre for Mathematics and Computer Science, Amsterdam, 1986.

[7] E. T. Whittaker and G. N. Watson, A Course of Modern Analysis, Fourth edition, Cambridge University Press, London, New York, 1927. 